\title{
CONCEPT OF PHOTONIC HOOK SCALPEL GENERATED BY SHAPED FIBER TIP WITH ASYMMETRIC RADIATION
}

\author{
Igor V. Minin ${ }^{1,2, *}$, Oleg V. Minin ${ }^{1,2}$, Yan-Yu Liu ${ }^{3}$, Cheng-Yang Liu ${ }^{3, * *}$ \\ ${ }^{1}$ Tomsk Polytechnic University, Tomsk, Russia \\ ${ }^{2}$ National Research Tomsk State University, Tomsk, Russia \\ ${ }^{3}$ Department of Biomedical Engineering, National Yang-Ming University, Taipei City, Taiwan \\ *E-mail: prof.minin@gmail.com \\ **E-mail: cyliu66@ym.edu.tw
}

Structured light have made deep impacts on modern biotechnology and clinical practice, with numerous optical systems and lasers currently being used in medicine to treat disease. We demonstrate a new concept of fiber-based optical hook scalpel. The subwavelength photonic hook is obtained in the vicinity of a shaped fiber tip with asymmetric radiation. A $1550 \mathrm{~nm}$ continuouswave source, commonly used for medical imaging, has been required. Photonic hook with a lateral feature size less than the half-wavelength is achieved using a hemispherical shaped fiber tip with metallic mask. This breakthrough is carried out in ambient air by using a $4-\mu \mathrm{m}$-diameter fiber with a shaped tip. A good correlation is observed between the computed intensity distribution of photonic hook and the tip sizes. Photonic hook generated with a shaped fiber tip, easier to manipulate, shows far-reaching benefits for potential applications such as ophthalmic laser surgery, super-resolution microscopy, photolithography, and material processing.

Keywords: photonic hook, optical scalpel, fiber.

Introduction

The use of light in medicine began thousands of years ago. The first laser light interaction with tissue was, probably, studied experimentally by Milton M. Zaret et al in 1961, who exanimated photocoagulation in the retina [1]. Three years later C. Kumar N. Patel invented the carbon dioxide gas laser in 1964 [2]. Later this gas laser was used for the first time to perform soft tissue surgery. L. Goldman and R.G. Wilson studied the destruction of skin lesions in 1964 [3]. Today one of the key elements of this medical device is a laser scalpel connected with an optical fiber to add of 
flexibility [4]. Different focusing devices are used on a fiber end to improve the light radiation exposure efficiency [5-7]. Focusing of multimodal light beams may be used in ultraprecise laser procedures [8] in the brain and eye with help of chains of dielectric microspheres assembled inside the cores of hollow waveguides [9,10]. It was shown that near a shaped optical fiber photonic jets (PJs) can be obtained at wavelength of $1064 \mathrm{~nm}$ [11,12]. Such structures [8-11] can focus laser beam into tissue in 'contact mode' with the tip of the optical scalpel and can ablate the tissue. Moreover, such laser scalpels were used for dissecting and removing fibrotic membranes from the retina surface [13]. In ophthalmic laser surgery, for example, the micro-hook needles are used to remove deposits from the retina for proliferative diabetic retinopathy [14]. The metal micro-hook scalpel has been used in neurosurgery for a long time due to its little contact with adjacent tissues [15].

The idea of application of recently discovered [16] a photonic hook (PH) effect $[17,18]$, based on dielectric Janus particle, as the ultraprecise laser scalpel for the first time was offered in [19]. It has recently been shown, that a PH in free space can also be obtained by asymmetric illumination of symmetric microparticle [20,21].

In this paper, we propose a fiber-based laser hook scalpel concept which the curvature and length of need to be carefully balanced. In this study, we report on the formation of a curved optical hook created by fiber with mesoscale hemispherical particle with partially blocking fiber tip by an amplitude mask height $h$. The key parameters of the PH such as lateral full width at half maximum (FWHM), curvature, and maximal field intensity depending on the variation of the amplitude mask height are studied systematically. The wavelength of the laser beam is $1550 \mathrm{~nm}$. The materials of the fiber and hemispherical tip are pure silica with refractive index of 1.44 [22]. The diameter of multimode optical fiber is $4 \mu \mathrm{m}$.

\section{Simulation model}

The objective lens is used to focus the laser beam into the fiber core. The metal mask is made of aluminum. In this study, we conduct finite-difference time-domain (FDTD) simulations [23] to analyze the effect on PH structure, illustrated in Figure 1. The simulation domain is set to be $3 \mu \mathrm{m}$ $\times 10 \mu \mathrm{m}$, the grid size of the FDTD-mesh is $30 \mathrm{~nm}$, and external boundary conditions are the perfectly matched layer for diminishing the effect of reflected lightwave at the boundary. 


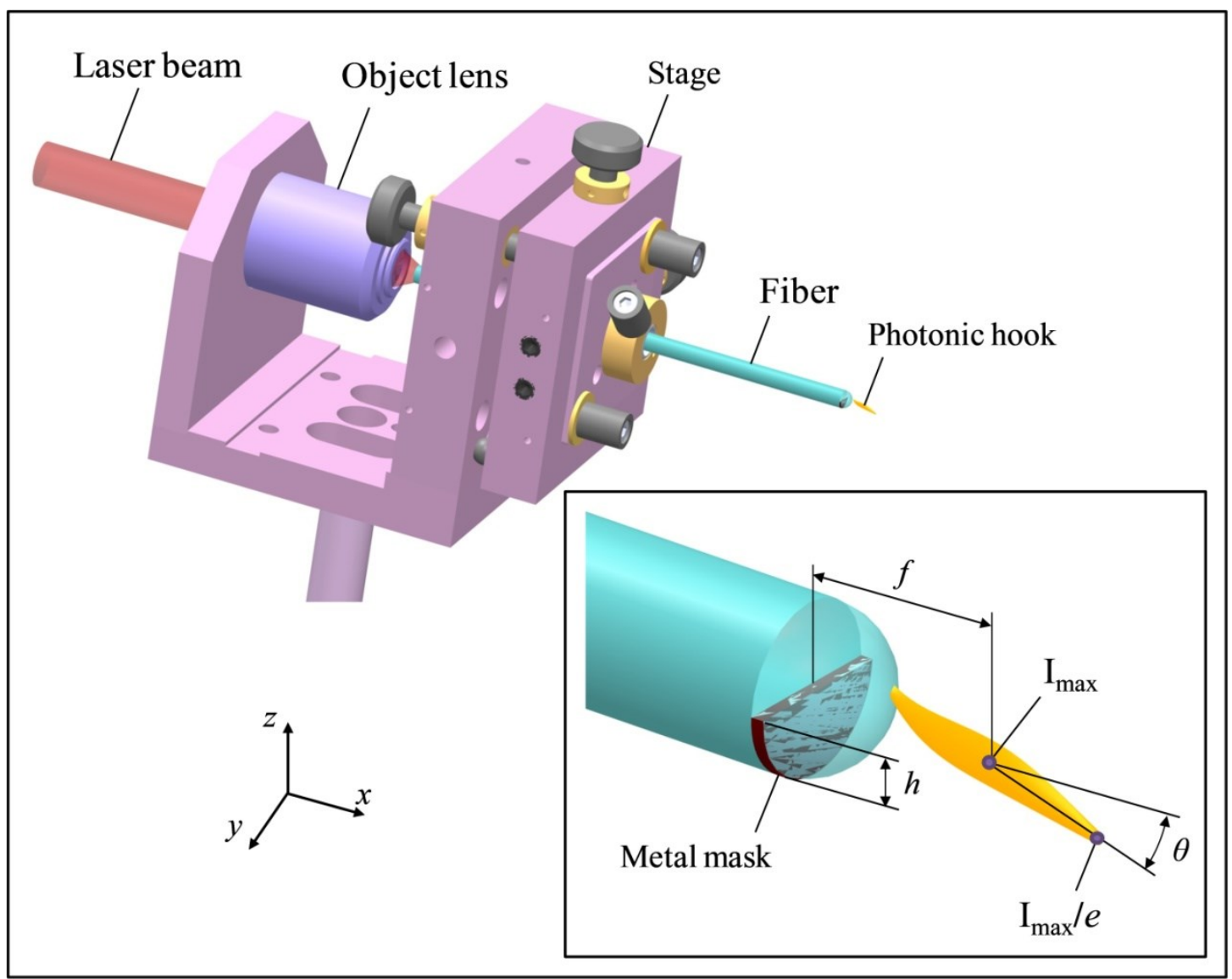

Figure 1. Schematic diagram of the fiber tip with metallic mask. The insert indicates the definition of the photonic hook.

Results of simulations

To illustrate the effect of the irradiating beamwidth on the PH formation, Figure 2 shows the power flow patterns of the PHs formed by fiber with hemispherical particle tip with different mask heights. The width of the irradiating beam is regulated by the height of the amplitude mask. It can be clearly seen that the PH curvature and length changes as the mask height increases. However, the $\mathrm{PH}$ position moves inside the fiber tip when the height of the amplitude mask is more than $0.35 d$. Following to [20-21] the physics of the curved PNJ formation can be explained as follows. The refractive index of fiber and width of illumination determines the angle of refraction on an interface via the generalized Snell's law [24]. Part of the illuminating beam inside the fiber and determined by the mask height, is diffracted first on the metall mask. Than the light beam inside the fiber is refracted when it exits from the shadow surface of the fiber tip (see Fig. 1). If the width of the illuminating beam is less than the fiber diameter, the components of the wave vector $\mathrm{K} \|$ do not cancel each other inside the fiber through the local destructive interference [20-21]. The wave 
vector $\mathrm{K} \|$ is relative to the axis of symmetry of the fiber, which creates the PH curvature profile. On the other hand, the components of the wave vector $\mathrm{K} \perp$ determine the length of the $\mathrm{PH}$ along the propagation direction. Therefore, the local optical fields interfere inside the fiber and then the $\mathrm{PH}$ can be generated outside the fiber tip depending on the width of the illuminating beam.

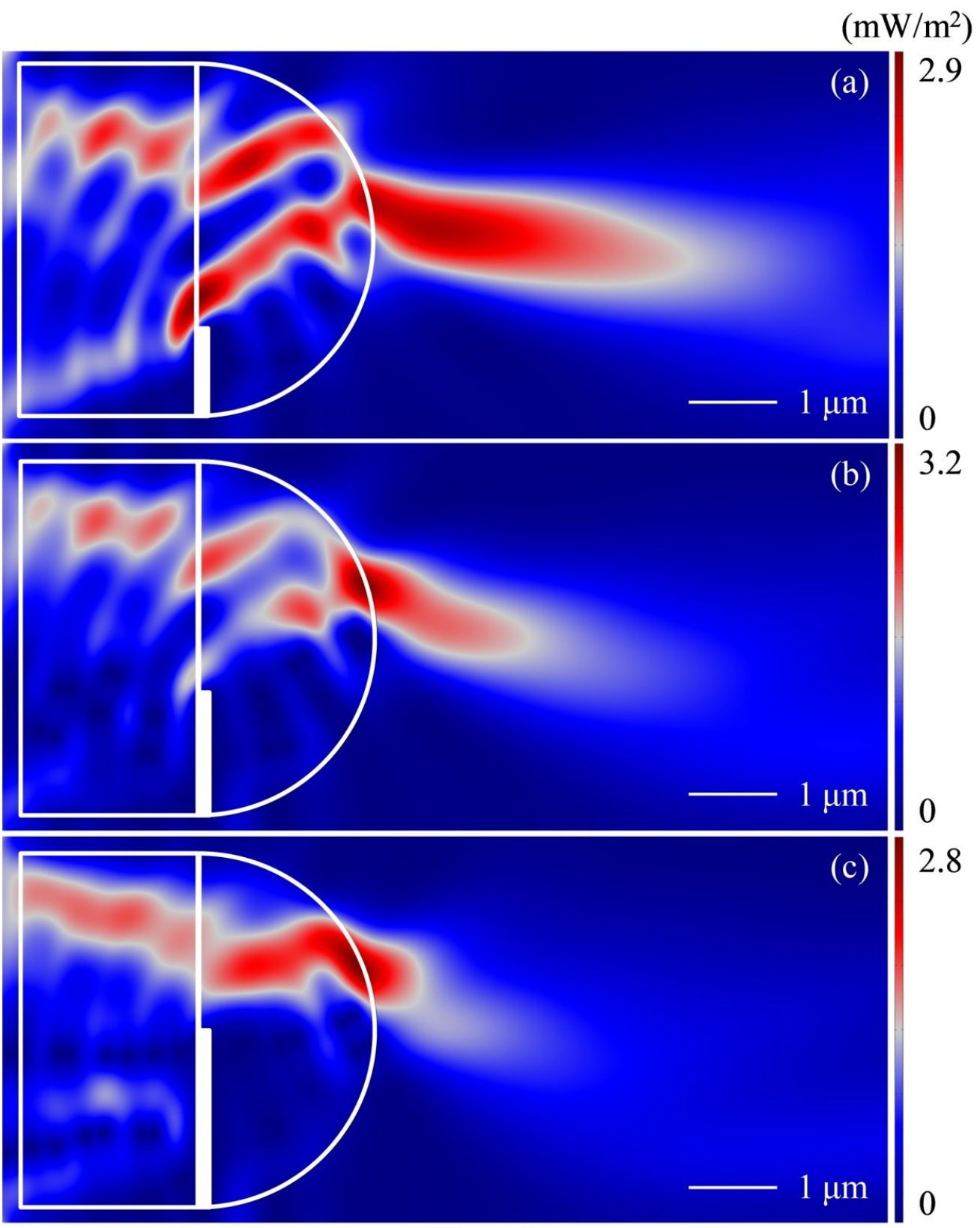

Figure 2. Power flow patterns of the photonic hooks formed by fiber tip with metallic mask at (a) $h=0.25 d$, (b) $h=0.35 d$, and (c) $h=0.5 d$, excited by the fundamental mode of the fiber.

The difference between the intensity distributions generated at different mask height $h$ values can be compared by the field intensity profiles. Figure 3 shows the normalized field intensity profiles of 
the PHs along the transverse direction, measured at maximal peak intensity plane. It can be seen that FWHM of the PH at $h=0.25 d$ is $0.55 \lambda$. For other values of the mask height it could be noted that maximal field intensity peak is located inside the hemispherical tip of the fined with refractive index $n=1.44$. The FWHMs at $h=0.35 d$ and $h=0.5 d$ are $0.42 \lambda$ and $0.4 \lambda$, respectively.

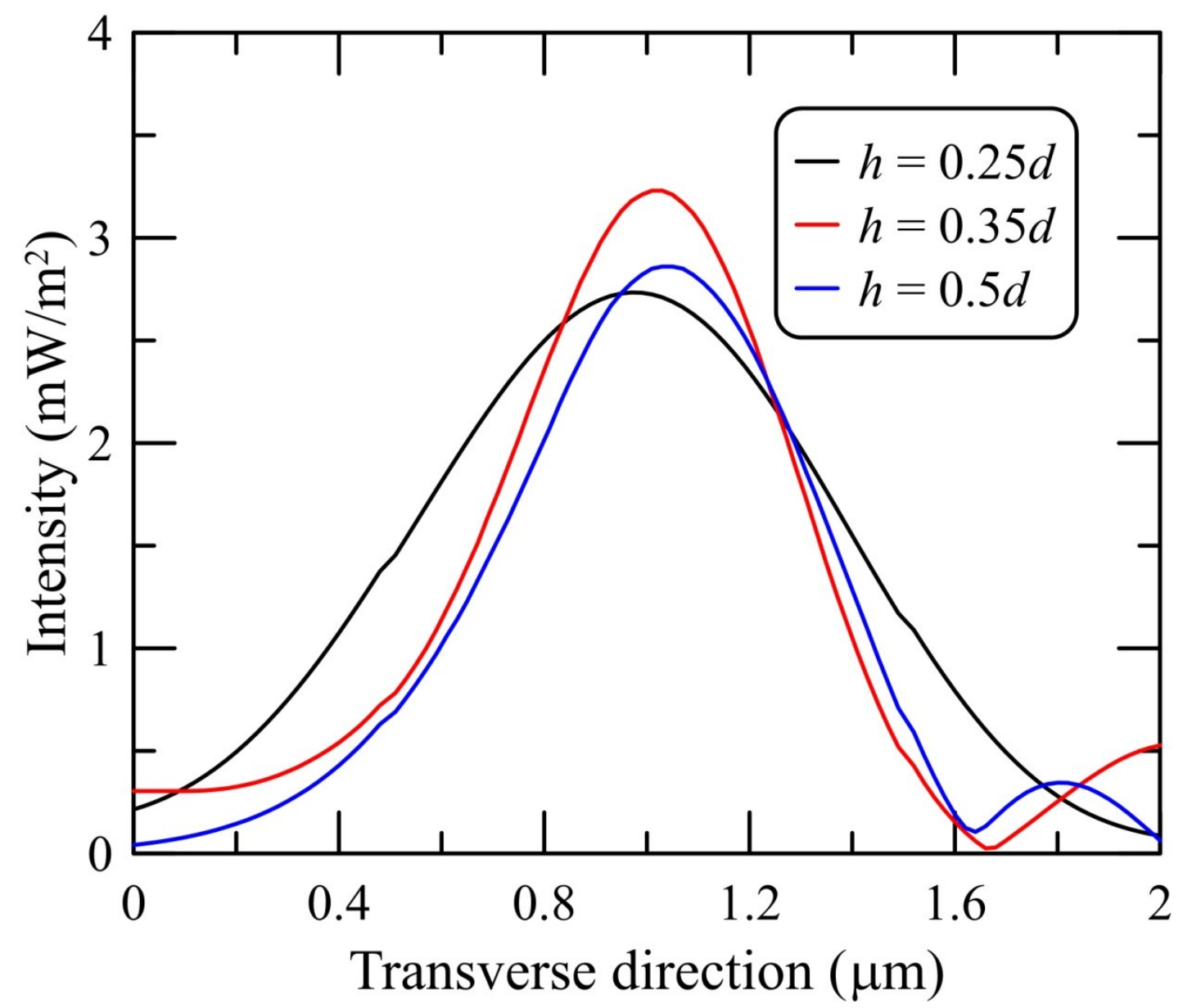

Figure 3. Intensity profiles of the photonic hooks for fiber tip along transverse direction.

The photonic hooks key parameters (focal length, FWHM, and tilt angle) as a function of the mask height, which define the curvature of the PH [17-18], are shown in Figure 4. For evaluating the tilt angle and the FWHM of the PHs [25], we find the inflection point (Imax) in the simulation images. The FWHM is defined as the double distance perpendicular to the propagation direction between Imax and the half-maximum point. If the whole PH is generated outside the fiber tip, the end and start points are located at 1/e Imax on the left and right sides of Imax along the PH. If the PH distributes across the fiber tip boundary, the start point is the intersection point of the $\mathrm{PH}$ and the fiber tip boundary. The left (start-inflection points) and right (end-inflection points) PH arms 
relative to the direction of radiation incidence are obtained as shown in Fig. 1. The tilt angle $\theta$ is identified as the supplementary angle between the left and the right PH arms.

The dash line in Fig. 4(a) corresponds to the boundary of hemispherical fiber tip. It can be clearly seen that with mask height less than $h \leqq 0.32 d$ the maximal field intensity position along the PH is out of fiber tip. The diffraction limited PH (FWHM $\leqq \lambda / 2)$, equal to FWHM=0.45 mask height $h=0.32 d$ with focal length of $\mathrm{F}=1.3 \lambda$ and tilt angle about 25 degree. Also one can see the mask height has a significant influence on the curvature of the $\mathrm{PH}$, as expected. 

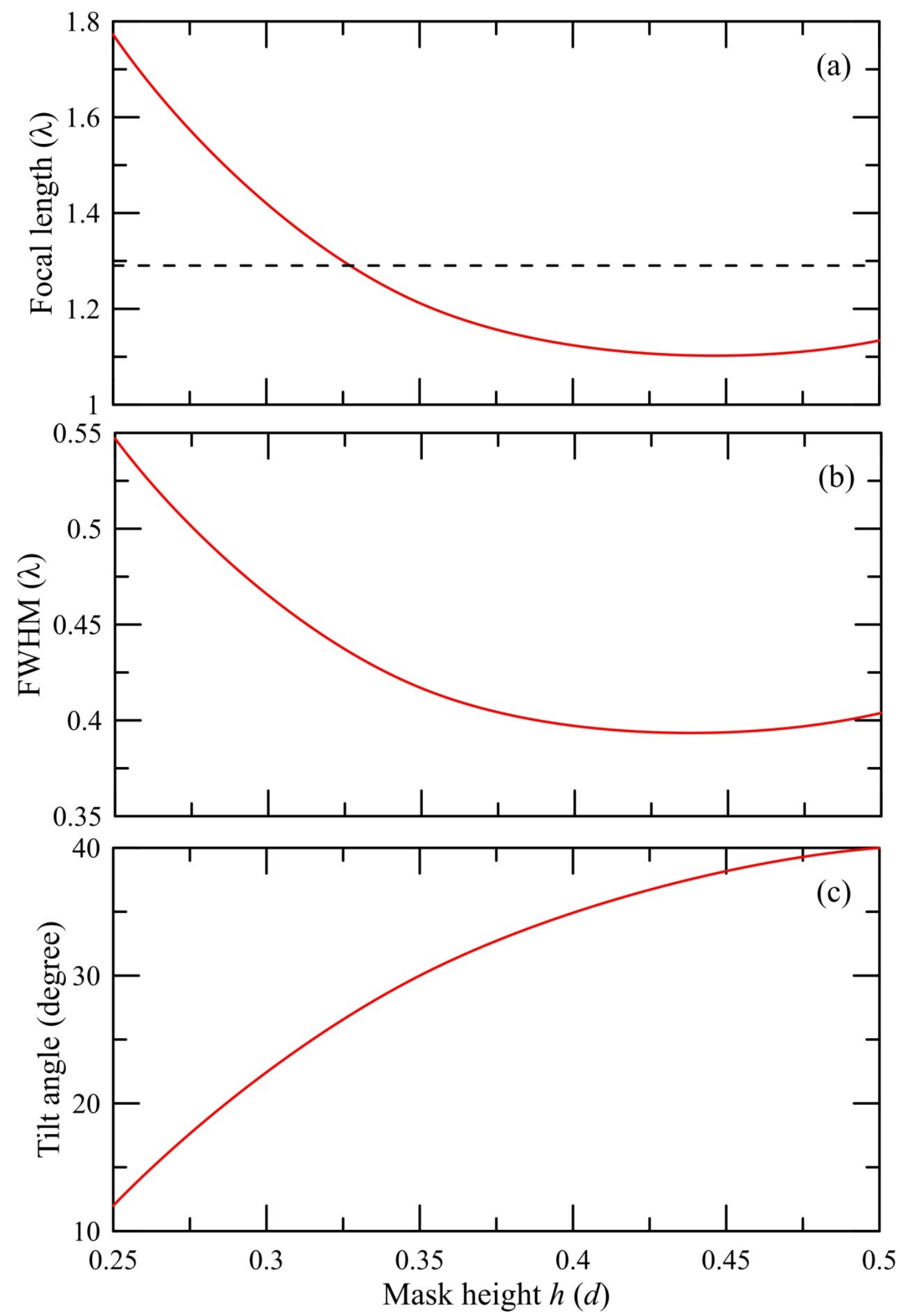

Figure 4. Key parameters as a function of the mask height for photonic hooks: (a) focal length, (b) FWHM, and (c) tilt angle. The dash line in Fig. 4(a) is the boundary of hemispherical tip. 


\section{Conclusions}

In conclusion, we have described the concept of optical hook fiber scalpel based on photonic hook phenomenon. Different laser scalpel configurations with varying metal mask dimension were successfully simulated demonstrating the concept of optical fiber-based hook scalpel. We have shown that the spatial shape and curvature of the PH with FWHM below the diffraction limit can be simply tailored by varying the metal mast inserting into the fiber tip. The correct combination of the illuminating beamwidth vs mask height enables the efficient beam shaping and directionality of the $\mathrm{PH}$ launching. The orientation of this $\mathrm{PH}$ with a tissue contact strongly determines the heated area. Thus, fibers based photonic hook enable to optimize tissue cutting modes due to controllable curved shape of the field localization area. Using the FDTD technique, we have demonstrated the key characteristics of the PH optical scalpel formed in the vicinity of the hemispherical fiber tip with metallic mask. In practical applications, a gel-like medium may be used to mimic the optical properties of a biological tissue [26,27]. Photonic hook is likely to make a significant contribution is the medical field and more precisely the field of surgery with a minimally traumatic. Sapphire waveguides are also of potential interest, but their drawback are a high refractive index and fragility of the material $[28,29]$.

The glancing angle deposition in sputtering system can be used to produce the fiber tip with metal mask [20]. The refractive index of a metal mask is varied by using different metal targets during deposition. We are currently building glancing angle deposition system for coating metal mask on the flat surface of the fiber tip. The further investigations of such instruments should be done. Moreover, the results of this work may be of interest for surface texturing [31-33] and nanostructuring, biosensing [34], fiber-based sub-wavelength optical micromanipulation [35-37], improvement of data storage, engraving, micro-welding, to name a few. This will be investigated.

Disclosures and Contributions. The authors have no relevant financial interests in this article and no potential conflicts of interest to disclose. Contributions: I.M. and O.M initiated the ideas of this work and directed the project, wrote the manuscript. Y.Y.L. and C.Y.L. made simulations, I.M., O.M., and C.Y.L. analyzed results. All authors contributed to discussions and commented on the manuscript.

\section{Acknowledgments}

This study is funded by Ministry of Science and Technology of Taiwan under Grant Nos. MOST 108-2221-E-010-012-MY3 and MOST 109-2923-E-010-001-MY2, and Yen Tjing Ling Medical Foundation under Grant No. CI-109-24, and is partially supported by Russian Foundation for Basic 
Research (20-57-S52001). This work was also partially carried out within the framework of the Tomsk Polytechnic University.

References

1. M. M. Zaret, G. M. Breinin, H. Schmidt, H. Ripps, I. M. Siegel, L. R. Solon. Ocular Lesions Produced by an Optical Maser (Laser). Science, 134 (3489), 1525-1526 (1961).

2. Patel, C. K. N. (1964). "Continuous-Wave Laser Action on Vibrational-Rotational Transitions of $\mathrm{CO}_{2}$ ". Physical Review. 136(5A): A1187-A1193.

3. Goldman, L. Wilson, R. G. Treatment of basal cell epithelioma by laser radiation. JAMA 189, 773-775 (1964).

4. Keiser, G., Xiong, F., Cui, Y., and Shum, P. P., Review of diverse optical fibers used in biomedical research and clinical practice, Journal of Biomedical Optics 19(8), 080902 (2014).

5. Stock K., Stegmayer T., Graser R., Förster W., Hibst R. Comparison of different focusing fiber tips for improved oral diode laser surgery. Lasers Surg Med 2012; 44(10): 815-823.

6. Freebody M. Fiber lasers at the cutting edge of surgery. Biophotonics 2013; 20(3).

7. M. C. Chiang, C. C. Huang, "Optically-guided scalpel with lightscattering module for carpal tunnel surgical procedure via minimally invasive surgery", Bio-medical Materials and Engineering, Vol. 26, No. S1, pp. S173-S179, 2015

8. P. Rol, and P. Niederer, "High-power laser transmission through optical fibers: Applications to ophthalmology," in Laser Applications in Medicine and Biology, M. Wolbarsht, Ed., 141-198, Plenum, New York (1991).

9. A. Darafsheh, A. Fardad, N. M. Fried, A. N. Antoszyk, H. S. Ying, and V. N. Astratov. Contact focusing multimodal microprobes for ultraprecise laser tissue surgery. Opt. Express, 2014; 19(4): 3440-3448.

10. T.C. Hutchens, A. Darafsheh, A. Fardad, A. N. Antoszyk, H. S. Ying, V. N. Astratov, N. M. Fried. Characterization of novel microsphere chain fiber optic tips for potential use in ophthalmic laser surgery, J. of Biomedical Optics, 17, 068004 (2012).

11. J. Zelgowski, A. Abdurrochman, F. Mermet, P. Pfeiffer, J. Fontaine, and S. Lecler. Photonic jet subwavelength etching using a shaped optical fiber tip. Opt. Lett., 41(9), 2073 (2016) 
12. A. Abdurrochman, J. Zelgowski, S. Lecler, F. Mermet, B. Tumbelaka and J. Fontaine. Photonic Jet Etching: Justifying the Shape of Optical Fiber Tip. AIP Conference Proceedings 1712, 030001 (2016).

13. D.X. Hammer et al:: Intraocular laser surgical probe for membrane disruption by laserinduced breakdown," Appl. Opt., vol. 36, pp. 1684-1693 (1997).

14. G. L. Spaeth, Ophthalmic Surgery: Principles and Practice, W. B. Saunders, Philadelphia, USA (1982).

15. S. Yang, J. Lu, D. Fu, D. Shang, F. Zhou, J. Liu and M. Cao. Effect of microscopically assisted decompression with micro-hook scalpel in the surgical treatment of ossification of the posterior longitudinal ligament. Journal of International Medical Research. 2019, Vol. 47(10) 5120-5129.

16. Minin, I.; Minin, O. Diffractive Optics and Nanophotonics. Resolution Below the Diffraction Limit; Springer International Publishing: Cham, Switzerland, 2015; doi:10.1007/978-3-31924253-8.

17. Yue L, Minin O V, Wang Z, Monks J, Shalin A and Minin I V 2018 Photonic hook: a new curved light beam Opt. Lett. 43771

18. Minin I V, Minin O V, Katyba G, Chernomyrdin N, Kurlov V, Zaytsev K, Yue L, Wang Z and Christodoulides D 2019 Experimental observation of a photonic hook Appl. Phys. Lett. 114031105

19. O. V. Minin, I. V. Minin, N. Kharitoshin. Microcubes Aided Photonic Jet Scalpel Tips for Potential Use in Ultraprecise Laser Surgery. 2015 International Conference on Biomedical Engineering and Computational Technologies (SIBIRCON), 28-30 Oct. 2015, p.18-21. DOI: 10.1109/SIBIRCON.2015.7361842

20. I. V. Minin, O. V. Minin, C.-Y. Liu, and H.-D. Wei, A. Karabchevsky. Simulation and experimental observation of tunable photonic nanojet and photonic hook upon asymmetric illumination of a mesoscale cylinder with mask. ArXiv: 2004.05911 (2020)

21. C.-Y. Liu; H.-J. Chung, O.V. Minin, I.V. Minin. Shaping photonic hook via well-controlled illumination of finite-size graded-index micro-ellipsoid. Journal of optics 22, 085002 (2020).

22. I. H. Malitson. Interspecimen comparison of the refractive index of fused silica, J. Opt. Soc. Am. 55, 1205-1208 (1965)

23. Taflove A and Hagness S 2005 Computational Electrodynamics: The Finite Difference Time Domain Method (Artech House) 
24. N. Yu, P. Genevet, M. Kats, F. Aieta, J. Tetienne, F. Capasso, and Z. Gaburro, "Light propagation with phase discontinuities: generalized laws of reflection and refraction," Science 334, 333-337 (2011).

25. Y. Geints, I. V. Minin, and O. V. Minin, "Tailoring 'photonic hook' from Janus dielectric microbar,” J. Opt. 22, 065606 (2020).

26. Tuchin, V. V., [Tissue Optics: Light Scattering Methods and Instruments for Medical Diagnostics, 3rd ed.], vol. PM 254, SPIE Press, Bellingham, WA (2015).

27. T. L. Troy and S. N. Thennadil. "Optical properties of human skin in the near infrared wavelength range of 1000 to $2200 \mathrm{~nm}$," Journal of Biomedical Optics 6(2), (2001).

28. G.M. Katyba, N.V. Chernomyrdin, I.N. Dolganova, A.A. Pronin, I.V. Minin, O.V. Minin, K.I. Zaytsev, V.N. Kurlov. Step-index sapphire fiber and its application in a terahertz nearfield microscopy. Proc. SPIE 11164, Millimetre Wave and Terahertz Sensors and Technology XII, 111640G (18 October 2019)

29. I. Dolganova, A. Zotov, I. Shikunova, K. Zaytsev, P. Aleksandrova, E. Mukhina, V. Kurlov, "Experimental study of pointed sapphire needles for interstitial laser therapy," Proc. SPIE 11458, Saratov Fall Meeting 2019: Laser Physics, Photonic Technologies, and Molecular Modeling, 114580E (9 April 2020)

30. N. Wakefield, J. Sorge, M. Taschuk, L. Bezuidenhout, M. Brett, J. Sit, Control of the principal refractive indices in biaxial metal oxide films, J. Opt. Soc. Am. A 28, 1830-1840 (2011).

31. Y. F. Lu, L. Zhang, W. D. Song, Y. W. Zheng and B. S. Luk'yanchuk, Laser writing of a subwavelength structure on silicon (100) surfaces with particle-enhanced optical irradiation. J. Exp. Theor. Phys. Lett. 72(9), 457-459 (2000).

32. R. Pierron, S. Lecler, J. Zelgowski, P. Pfeifier, F. Mermet, and J. Fontaine, "Etching of semiconductors and metals by the photonic jet with shaped optical fiber tips," Applied Surface Science, vol. 418, Part B, pp. 452-455, 2017.

33. T. Uenohara, Y. Takaya, Y. Mizutani. Laser micro machining beyond the diffraction limit using a photonic nanojet. CIRP Annals - Manufacturing Technology, 66(1), 491-494 (2017).

34. C. Credi, O. Bibikova, C. Dallari, B. Tiribilli, F. Ratto, S. Centi, R. Pini, V. Artyushenko, R. Cicchi, Francesco S. Pavone, "Disposable and versatile optical sensors for SERS analysis of liquid samples by fiber-based spectroscopy," Proc. SPIE 11075, Novel Biophotonics Techniques and Applications V, 110750V (22 July 2019);

35. I.V. Minin, C.-Y. Liu, Y. E Geints, O. V. Minin. Recent advantages in Integrated Photonic Jet-Based Photonics. Photonics 2020, 7(2), 41 
36. Y. Lou, D, Wu, Y, Pang. Optical Trapping and Manipulation Using Optical Fibers. Advanced Fiber Materials 1, 83-100 (2019)

37. X. Zhao, N. Zhao, Y. Shi, H. Xin and B. Li. Optical Fiber Tweezers: A Versatile Tool for Optical Trapping and Manipulation. Micromachines 11, 114 (2020) 